\title{
Cosmic rays in magnetized intracluster plasma
}

\author{
L. Feretti ${ }^{1}$, A. Bonafede ${ }^{1}$, G. Giovannini ${ }^{1,2}$, F. Govoni ${ }^{3}$ \& M. Murgia ${ }^{3}$ \\ ${ }^{1}$ Istituto di Radioastronomia INAF, Via Gobetti 101, 40129 Bologna, Italy \\ ${ }^{2}$ Dipart. Astronomia, Univ. Bologna, Via Ranzani 1, 40127 Bologna, Italy \\ ${ }^{3}$ Oss. Astronomico Cagliari, Loc. Poggio dei Pini, Strada 54, 09012 Capoterra (CA), Italy
}

\section{Magnetic Fields in Clusters of Galaxies}

A breakthrough in the studies of magnetic fields in clusters of galaxies has been reached in recent years from the analysis of the Rotation Measure of sources seen through the magnetized cluster medium (Govoni \& Feretti 2004). The results obtained can be summarized as follows: (i) magnetic fields are present in all clusters; (ii) at the center of clusters undergoing merger activity the field strenght is around $1 \mu \mathrm{G}$, whereas at the center of relaxed cooling core clusters the intensity is much higher $(\sim 10 \mu \mathrm{G})$; (iii) a model involving a single magnetic field coherence scale is not suitable to describe the observational data, because of different scales of field ordering and tangling.

Assuming a magnetic field power spectrum: $\left|B_{\kappa}\right|^{2} \propto \kappa^{-n}$ (Murgia et al. 2004), the range of spatial scales is found between $30-500 \mathrm{kpc}$ and the spectral index $n$ is in the range $2-4$ (note that in A2255, Govoni et al. 2006 obtain a flatter index at the center, and a steeper index at the periphery, likely due to different turbulence development).

In addition, the cluster magnetic field intensity shows a radial decline linked to the thermal gas density $\mathrm{n}_{e}$ as $B \propto \mathrm{n}_{e}^{x}$. A trend with $x=1 / 2$ is expected if the $\mathrm{B}$ field energy scales as the thermal energy, while $x=2 / 3$ if the B field results from a frozen-in field during the cluster collapse. The values of $x$ derived so far are in the range $0.5-1$.

\section{Diffuse Radio Emission}

Magnetic fields at the $\mu \mathrm{G}$ level in the intracluster plasma are illuminated by cosmic rays, which give rise to diffuse cluster radio emission of synchrotron origin. While magnetic fields are ubiquitous in clusters, the radio emitting electrons are currently not known to be present in all clusters, although their presence is revealed in several conditions (merging and relaxed clusters), at different cluster locations (center, periphery, intermediate distance), on very different scales $(100 \mathrm{kpc}$ to $>\mathrm{Mpc}$ ), and generally is related to a high degree of magnetic field ordering. Most spectacular examples of diffuse radio emission are the giant radio halos and relics detected in merging clusters. Several giant double relics, located on opposite side with respect to the cluster center, are presently known (e.g. Bonafede et al. 2009, van Weeren et al. 2009). Radio halos of smaller size have also been detected, as well as mini-halos and small relics in cooling core clusters. New halos have been detected in A851, A1213, A1351, A1995, A2034 and A2294 (Giovannini et al. 2009, also Giacintucci et al. 2009 for A1351). Among them, the cluster A1213 is remarkable because its X-ray luminosity is about 10 times weaker than that associated with clusters hosting radio halos (as derived so far).

All diffuse radio sources have in common the very steep radio spectra, implying that the radiating particles have short lifetimes, and need to be reaccelerated.

\section{Radio - X-ray connection in radio halos}

The properties of radio halos are linked to the properties of the host clusters (Cassano et al. 2006, Giovannini et al. 2009), in particular: a) the radio power of both small and giant halos correlates with the cluster X-ray luminosity (i.e. gas temperature and total mass); b) the radio spectra of halos are affected by the cluster temperature, being flatter in hotter clusters, and in 

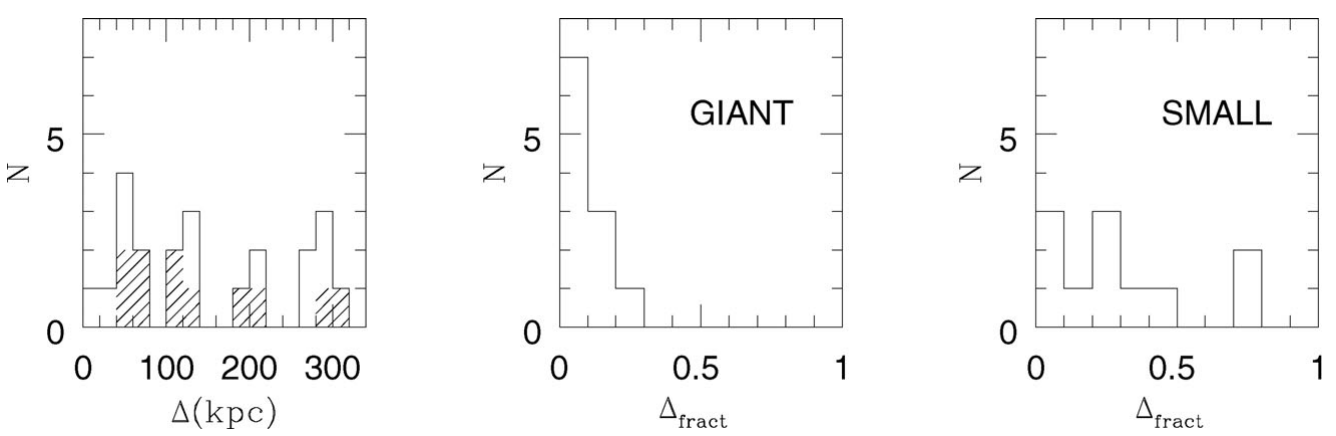

Figure 1. Left panel: Values of the offset $\Delta$ between the radio and X-ray centroids in kpc. The dashed area refers to giant halos. Middle and Right panels : Fractional offset ( $\Delta$ /radio halo size) for giant halos (size $\geqslant 1 \mathrm{Mpc}$ ) and small halos (size $<1 \mathrm{Mpc}$ ), respectively.

hotter cluster regions (Orrú et al. 2007); c) in a number of well-resolved clusters, a point-topoint spatial correlation is observed between the halo radio brightness and the cluster X-ray brightness (Govoni et al. 2001).

A step forward is to check whether the last property is common to all radio halos, i.e. whether radio halos are generally distributed as the $\mathrm{X}$-ray thermal gas. Using a sample of clusters having good radio and X-ray data, we have analysed the position of the radio halo with respect to that of the X-ray gas distribution. The left panel of Fig. 1 shows that both giant and small radio halos can be significantly shifted, up to hundreds kpc, with respect to the centroid of the host cluster. To highlight radio halos with the most pronounced asymmetric distribution, we have then derived the ratio between the radio-X-ray offset and the halo size. From the middle and right panels of Fig.1, we deduce that halos can be very asymmetric with respect to the X-ray gas distribution, and this becomes more relevant when halos of smaller size are considered. A possibility is that the asymmetry in the structure originates by magnetic field fluctuations as large as hundreds of kpc, as suggested by Vacca et al. (2009) on the basis of magnetic field modeling.

\section{References}

Bonafede, A., Giovannini, G., Feretti, L., Govoni, F., \& Murgia, M., 2009, A\&A 494, 429 Cassano, R., Brunetti, G., \& Setti, G., 2006 MNRAS 369, 1577

Giacintucci, S., Venturi, T., Cassano, R., et al., 2009, ApJl in press, eprint arXiv:0909.0437

Giovannini, G., Bonafede, A., Feretti, L., et al., 2009, A\&A in press, eprint arXiv0909.0911

Govoni, F., Enßlin, T. A., Feretti, L., \& Giovannini, G., 2001, A\&A 369, 441

Govoni, F. \& Feretti, L., 2004, Int. J. Mod. Phys. D, Vol., 13, 1549

Govoni, F., Murgia, M., Feretti, L., et al., 2006, A\&A 460, 425

Murgia, M., Govoni, F., \& Feretti, L. , 2004, A\&A 424, 429

Orrú, E., Murgia, M., Feretti, L., et al., 2007, A\&A 467, 943

Vacca V., Murgia, M., Govoni, F., et al., 2009, A\&A, Submitted

van Weeren, R. J., et al. 2009, A\&A in press, eprint arXiv0908.0728 